\title{
Processo maturacional, insatisfação corporal e comportamento alimentar inadequado em jovens atletas
}

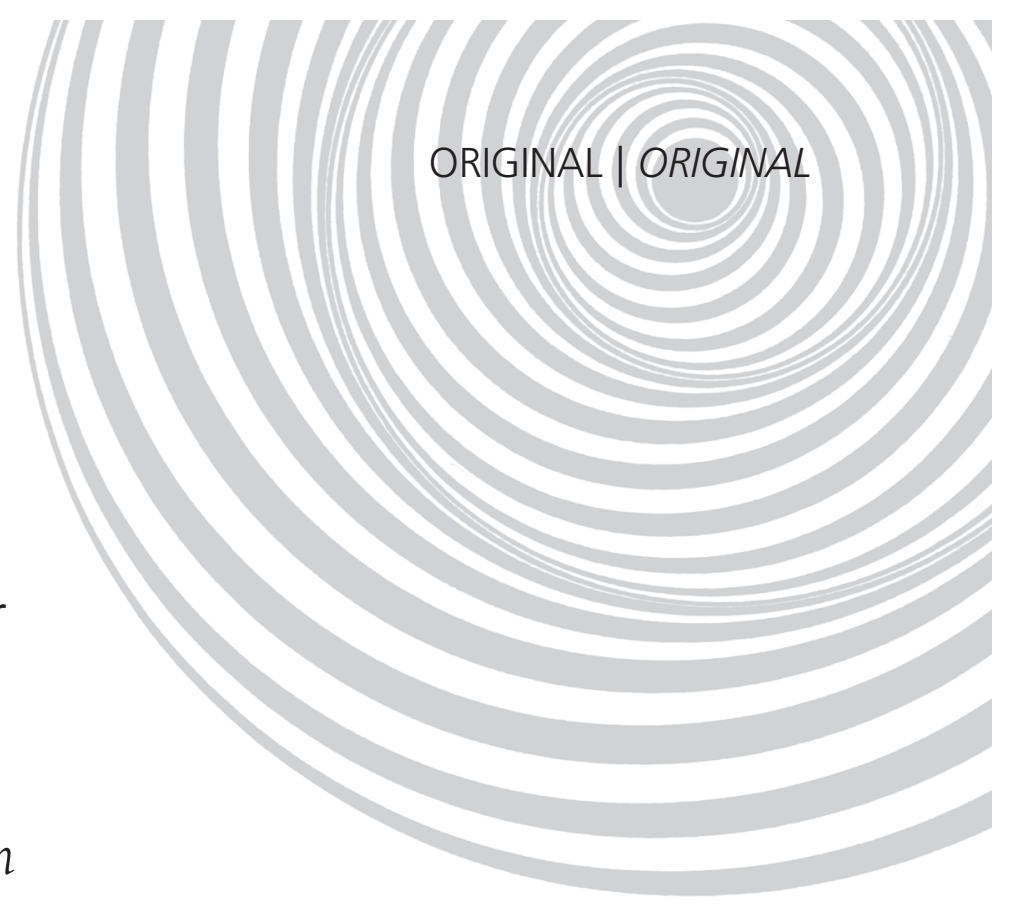

\author{
Maturation process, body dissatisfaction \\ and inappropriate eating behavior \\ in young athletes
}

Leonardo de Sousa FORTES ${ }^{1}$

Sebastião de Sousa ALMEIDA²

Maria Elisa Caputo FERREIRA'

RE S U M O

\section{Objetivo}

O objetivo deste estudo foi analisar a associação entre o processo maturacional, a insatisfação corporal e o comportamento alimentar inadequado, de acordo com o sexo, em jovens atletas.

\section{Métodos}

Participaram da pesquisa 580 indivíduos, de ambos os sexos, pertencentes a diferentes modalidades esportivas. Foram avaliados a maturação sexual, a maturação somática, a insatisfação corporal e o comportamento alimentar inadequado, por meio dos Critérios de Tanner, banco de Lohman, Body Shape Questionnaire e Eating Attitudes Test, respectivamente. O percentual de gordura foi estimado pela medição das dobras cutâneas, e a aferição de peso e de estatura foi utilizada para calcular o índice de massa corporal. Realizaram-se modelo Univariado de Covariância, regressão logística binária e regressão linear múltipla para análise dos dados.

\section{Resultados}

Os resultados demonstraram diferenças estatisticamente significativas $(p<0,05)$ na insatisfação corporal entre os estágios maturacionais. Além disso, os meninos pré-púberes e púberes apresentaram maior probabilidade de insatisfação corporal em relação aos atletas pós-púberes $(p<0,05)$, e o modelo de regressão logística mostrou associação entre os estágios maturacionais e o comportamento alimentar inadequado apenas no sexo masculino $(p<0,05)$. Nas meninas, o processo maturacional explicou em $9 \%$ e $7 \%$ a variância da insatisfação corporal $(p<0,05)$ e comportamento alimentar inadequado $(p<0,05)$, respectivamente.

\footnotetext{
${ }^{1}$ Universidade Federal de Juiz de Fora, Faculdade de Educação Física e Desportos, Laboratório de Estudos do Corpo. R. José Lourenço Kelmer, s/n., Campus Universitário, São Pedro, 36036-900, Juiz de Fora, MG, Brasil. Correspondência para/Correspondence to: LS FORTES. E-mails: <leosousafortes@gmail.com>; <leodesousafortes@hotmail.com>.

2 Universidade de São Paulo, Faculdade de Filosofia, Ciências e Letras de Ribeirão Preto, Departamento de Psicologia, Laboratório de Nutrição e Comportamento. Ribeirão Preto, SP, Brasil.
} 
576 | LS FORTES et al.

\section{Conclusão}

Constatou-se que a maturação biológica esteve associada ao comportamento alimentar inadequado somente entre jovens atletas do sexo masculino.

Termos de indexação: Comportamento alimentar. Desenvolvimento humano. Imagem corporal.

\section{A B S T R A C T}

\section{Objective}

The purpose of this study was to analyze the association between maturation process, body dissatisfaction and inappropriate eating behavior in young athletes according to gender.

\section{Methods}

Five-hundred eighty individuals of both genders practicing different sports were surveyed. Sexual maturation, somatic maturation, body dissatisfaction and inappropriate eating behavior were assessed by the Tanner, stock Lohman, Body Shape Questionnaire and Eating Attitudes Test, respectively. Percentage of fat was estimated by skinfold thickness and weight and height were measured for calculating the body mass index. Statistical treatment included univariate analysis of covariance, binary logistic regression and multiple linear regression.

\section{Results}

The results demonstrated statistically significant differences $(p<0.05)$ in body dissatisfaction among maturation stages. In addition, prepubescent and pubescent boys were more likely to be dissatisfied compared with post-pubescent athletes $(p<0.05)$. Furthermore, the logistic regression model showed an association between inappropriate eating behavior maturation stages only in males $(p<0.05)$. In females, the maturation process explained in $9 \%$ and $7 \%$ of the sample, respectively, the variation in body dissatisfaction $(p<0.05)$ and inappropriate eating behavior $(p<0.05)$.

\section{Conclusion}

Biological maturation was associated with inappropriate eating behavior only in young male athletes.

Indexing terms: Feeding behaviors. Human development. Body image.

\section{N T R O D U Ç Ã O}

A adolescência é um período compreendido entre 10 e 19 anos de idade ${ }^{1}$. Nessa fase, o indivíduo busca integrar-se à prática esportiva a fim de otimizar seu rendimento físico ${ }^{2,3}$ e conquistar a condição de atleta, o que implica a participação em competições para conquistas e vitórias e tentativas de exceder os limites ${ }^{3,4}$. Além disso, nessa etapa, ocorrem diversas alterações morfológicas no corpo do ser humano. A maior parte dessas modificações é regulada por hormônios específicos liberados por glândulas como a hipófise anterior e a tireoide, o que constitui o processo maturacional ${ }^{5}$.

Em meninas, esse processo gera aumento de gordura corporal, enquanto, nos meninos, é mais comum encontrar maior massa magra com o avanço da idade ${ }^{5}$. O Pico de Velocidade de Crescimento Estatural (PVA) é conhecido como um dos principais marcadores maturacionais, ocorrendo por volta dos 12 anos em meninas e dos 14 anos nos meninos ${ }^{6}$. Esse evento maturacional promove um dismorfismo corporal entre algumas áreas corporais, tais como: diâmetro biacromial, diâmetro bicristal, razão entre comprimento de membros inferiores e altura troncocefálica, além do aumento de peso corporal que, somado às alterações morfológicas mencionadas, pode provocar nos adolescentes o descontentamento com o corpo ${ }^{2}$. O desgosto com a própria aparência física pode repercutir negativamente na construção da imagem corporal? .

A imagem corporal é um construto multidimensional que sofre influência de diversos fatores $^{8}$. Existem evidências que explicam a variância de componentes da imagem corporal a partir da etnia ${ }^{4}$, do nível de atividade física ${ }^{7}$ e dos níveis competitivos e econômicos ${ }^{3}$. No entanto, acredita- 
-se que o processo maturacional também possa influenciar a imagem corporal. Atribui-se ao conceito de imagem corporal a imagem mental que é formulada do próprio corpo ${ }^{2,9}$. A insatisfação corporal, por sua vez, refere-se ao descontentamento que se tem com o peso e a aparência física, e faz parte da dimensão atitudinal da imagem corporal $^{9}$. Parece que a insatisfação corporal em países ocidentais, como o Brasil, está mais associada ao ideal morfológico que a mídia costuma impor: magreza para as meninas e musculosidade para os meninos ${ }^{3,4}$. Desse modo, os jovens que não se adequarem a tais tendências corporais podem acentuar suas preocupações com a aparência física ${ }^{2,9}$. Já foram desenvolvidos estudos que avaliam as interferências de peso ${ }^{2}$, cultura, autoestima $^{10}$, sexo, etnia ${ }^{11}$, prática de atividade física ${ }^{12}$ e composição corporal ${ }^{8}$ sobre a insatisfação com o corpo. Entretanto, pesquisas que tentaram compreender a variância da insatisfação corporal provocada por medidas antropométricas não levaram em consideração as etapas do processo maturacional.

Sabe-se que a insatisfação corporal pode predispor o indivíduo ao comportamento alimentar inadequado ${ }^{13}$, que se manifesta, por exemplo, pela indução de vômitos, longos períodos de restrição alimentar, preocupação exagerada com os alimentos, uso de medicamentos para emagrecimento (laxantes, diuréticos e inibidores de apetite), entre outros ${ }^{14}$, e que traz sérios prejuízos à saúde. A prevalência desses comportamentos é alta no âmbito competitivo ${ }^{3,15}$, entretanto parece se manifestar de forma diferente entre os sexos. Alguns estudos comprovam sua maior frequência no público feminino ${ }^{16,17}$, recomendando analisar esses comportamentos segundo sexo e faixa-etária. Ademais, pesquisadores pontuam a existência de inúmeros fatores que predispõem o adolescente a tais hábitos ${ }^{4,16} \mathrm{e} \mathrm{um}$ deles refere-se ao processo maturacional ${ }^{2}$.

Salienta-se que, até o momento, estudos foram desenvolvidos somente comparando a insatisfação corporal e o comportamento alimen- tar antes e após a chegada da menarca no sexo feminino ou tentando comparar essas variáveis em distintos períodos etários ${ }^{18}$. Entretanto, tais pesquisas não se preocuparam em utilizar métodos confiáveis para identificar o período do processo maturacional, tais como: maturação esquelética, somática e/ou sexual ${ }^{19}$. Todavia, não foram encontrados estudos semelhantes com amostra do sexo masculino. De antemão, acredita-se que o processo maturacional possa contribuir para o desenvolvimento do comportamento alimentar inadequado, além de predispor adolescentes ao desgosto com a aparência física². Apesar dessas hipóteses, ainda não foi realizado nenhum estudo que analise essa associação ou a influência do processo maturacional sobre a insatisfação corporal e o comportamento alimentar em jovens atletas. Diante do pressuposto, o objetivo do presente estudo foi analisar a associação entre o processo maturacional, a insatisfação corporal e o comportamento alimentar inadequado segundo o sexo em jovens atletas.

Por conseguinte, algumas hipóteses foram formuladas levando-se em conta os aspectos morfológicos valorizados na cultura ocidental ${ }^{20}$, considerando as modificações na composição corporal provocadas pelo processo de maturação biológica do ser humano ${ }^{5}$. Espera-se que estágios pubertários mais avançados assumam maior risco para insatisfação corporal e comportamento alimentar inadequado em atletas femininas. Todavia, estima-se que meninos em estágios maturacionais iniciais apresentem maior risco para insatisfação corporal e comportamento alimentar inadequado. Por fim, pressupõe-se que a maturação biológica (sexual e somática) exerça influência significativa na insatisfação corporal e no comportamento alimentar em ambos os sexos.

\section{MÉ TO D OS}

Trata-se de um estudo transversal, com características de associação e comparação. A priori, as federações de várias modalidades espor- 
tivas e alguns clubes localizados nos estados da região Sudeste foram contatados e convidados por telefone ou e-mail a participar da pesquisa. No entanto, obtiveram-se respostas somente dos estados do Rio de Janeiro e de Minas Gerais. Sendo assim, a população da pesquisa representou atletas adolescentes com idade entre 10 e 19 anos, de ambos os sexos, residentes nos estados do Rio de Janeiro e de Minas Gerais. Segundo o Comitê Olímpico Brasileiro, no ano de 2010, essa população era de 24252 indivíduos. Realizou-se o cálculo amostral, considerando prevalência de $20 \%$ para comportamentos alimentares inadequados, com 95\% de intervalo de confiança, $4 \%$ de erro amostral e efeito de desenho de 1,4, totalizando 379 jovens. Todavia, por recomendações de Krentz \& Warschburger ${ }^{17}$, decidiu-se coletar o máximo de dados possível, além do tamanho amostral calculado. Nesse sentido, fica evidente a preservação da representatividade da amostra sobre a população da presente investigação, independentemente da quantidade de perdas no tamanho amostral. Somente foram incluídos na pesquisa sujeitos que apresentassem rotina de treinamento físico sistematizado, com frequência de três vezes por semana, com duração de uma hora, e que tivessem participado de competições oficiais. Desse modo, foram incluídos no estudo 620 atletas de ambos os sexos com tempo médio de treinamento de 3,19 (Desvio-Padrão-DP=1,08) anos de acordo com o esporte praticado; porém, 40 desses sujeitos foram excluídos da amostra por não responderem aos questionários em sua totalidade, e/ou não participarem da aferição de medidas antropométricas, além de apresentarem dados maturacionais incompletos, totalizando uma amostra final de 580 esportistas (Tabela 1). Para seleção da amostra, foi utilizado o método de amostragem casual simples ${ }^{20}$.

A pesquisa foi iniciada após aprovação do Comitê de Ética e Pesquisa em Seres Humanos da Universidade Federal de Juiz de Fora, com parecer $n^{\circ}$ 232/2010 (Protocolo n 2149.209.2010). O Termo de Consentimento Livre e Esclarecido, explicando todos os procedimentos da pesquisa e garantindo o anonimato aos participantes e total sigilo no tratamento dos dados, foi assinado pelos responsáveis e pelos próprios atletas.

\section{Medidas}

A avaliação da maturação sexual foi realizada por interposição dos Critérios de Tanner ${ }^{21}$ :

Tabela 1. Distribuição de frequência dos atletas segundo sexo e modalidade esportiva. Rio de Janeiro (RJ), Três Rios (RJ), Juiz de Fora (MG), Barbacena (MG), Belo Horizonte (MG), 2011.

\begin{tabular}{lcccc}
\hline Modalidade & \multicolumn{2}{c}{ Feminino } & \multicolumn{2}{c}{ Masculino } \\
\hline & FA (n) & FR (\%) & FA (n) & 2,2 \\
Atletismo & - & - & 10 & 12,1 \\
Basquetebol & 16 & 13,8 & 56 & 1,7 \\
Esgrima & - & - & 8 & 58,4 \\
Futebol & - & - & 271 & - \\
Ginástica Artística & 14 & 12,1 & - & 3,4 \\
Handebol & 20 & 17,2 & 16 & 3,0 \\
Judô & - & 19,0 & 14 & 9,1 \\
Natação & 22 & - & 42 & 3,4 \\
Polo Aquático & - & 4,3 & 16 & 1,1 \\
Saltos Ornamentais & 5 & 4,3 & 5 & 1,3 \\
Tae kwon do & 5 & - & 6 & 3,0 \\
Triathlon & - & 13,8 & 14 & 1,3 \\
Voleibol & 16 & 100,0 & 6 & 100,0 \\
\hline Total & 116 & & 464 & \\
\hline
\end{tabular}

FA: Frequência Absoluta; FR: Frequência Relativa. 
um método de autoavaliação que procura identificar o período do processo maturacional no qual 0 adolescente se encontra ${ }^{19}$, validado para a população brasileira por Matsudo \& Matsudo ${ }^{22}$. A classificação dos Critérios de Tanner é descrita da seguinte maneira: Estágio 1: Pré-púbere; Estágio 2: Púbere inicial; Estágio 3: Púbere intermediário; Estágio 4: Púbere avançado; Estágio 5: Pós-púbere.

A maturação somática busca estimar o PVA ${ }^{6}$. Para isso, é necessária a aferição única da estatura, do peso e da altura troncocefálica. $\mathrm{O}$ comprimento de membros inferiores é calculado pela diferença entre estatura e altura troncocefálica, bastando combinar essas variáveis com a idade cronológica, em equações propostas por Mirwald et al. ${ }^{6}$, segundo sexo do avaliado. As fórmulas fornecem resultados com pontuações negativas e positivas. Valor negativo significa que o sujeito ainda vai atingir o PVA, e valor positivo apresenta que o indivíduo já passou do PVA ${ }^{6}$. A classificação pode ser feita da seguinte maneira:

$<-1$ = pré-estirão de crescimento em estatura;

entre -1 e +1 = durante estirão de crescimento em estatura;

$>+1$ = pós-estirão de crescimento em estatura.

Para avaliar a insatisfação corporal, foi aplicado o Body Shape Questionnaire (BSQ): um teste de autopreenchimento, com 34 perguntas voltadas para a preocupação que o sujeito apresenta com seu peso e sua aparência física. A versão utilizada foi validada para adolescentes brasileiros $^{23}$. Devido ao fato de o BSQ não ter sido criado para o âmbito esportivo, decidiu-se seguir as recomendações de Filaire et al. ${ }^{10}$, calculando-se a consistência interna para a presente amostra. Identificaram-se, portanto, valores do alfa de Cronbach de 0,91 para meninas e de 0,92 para os meninos. O escore do BSQ é atribuído à soma dos seus itens, que classifica níveis de insatisfação a respeito do corpo: $<80$ pontos - livre de insatisfação corporal; entre 80 e 110 - leve insatisfação; entre 110 e 140 - insatisfação moderada; e pon- tuações acima de 140 - grave insatisfação corporal.

O comportamento alimentar inadequado foi avaliado por meio do Eating Attitudes Test (EAT-26): um questionário com 26 questões que avalia recusa patológica alimentar, preocupação exacerbada com aparência física, comportamentos purgativos, influência do ambiente na ingestão alimentar e autocontrole sobre os alimentos. A versão utilizada para o sexo feminino foi validada por Bighetti et al. ${ }^{14}$. Em adolescentes do sexo masculino, a versão aplicada foi a de Fortes et al. ${ }^{24}$. Assim como realizado com o BSQ, calculou-se o alfa de Cronbach para o EAT-26, e obtiveram-se valores de 0,89 para o sexo feminino e de 0,92 para o masculino. A pontuação do EAT-26 é obtida pela soma dos seus itens. Escore igual ou maior que 20 representa indivíduos com comportamentos alimentares de risco para transtornos alimentares. Existem seis opções de respostas que variam de 0 a 3 pontos (sempre $=3$, muitas vezes $=2$, frequentemente $=1$, poucas vezes $=0$, quase nunca $=0$ e nunca $=0$ ). A única questão que apresenta pontuação em ordem invertida é a 25 (sempre $=0$, muitas vezes $=0$, frequentemente $=0$, poucas vezes $=1$, quase nun$\mathrm{ca}=2$ e nunca $=3$ ).

As medidas antropométricas coletadas foram massa corporal, estatura e dobras cutâneas (subescapular e triciptal). Utilizaram-se os procedimentos descritos pela The International Society for the Advancement of Kinanthropometry ${ }^{25}$. Utilizou-se auxílio de uma balança eletrônica portátil da Tanita ${ }^{\circledR}$, com precisão de $0,1 \mathrm{~kg}$, para mensurar o peso, e um estadiômetro portátil, com precisão de $0,1 \mathrm{~cm}$, da Tonelli® ${ }^{\circledR}$, para aferir a estatura. Obteve-se o Índice de Massa Corporal (IMC) por meio da razão entre massa corporal $(\mathrm{kg})$ e quadrado da estatura $(\mathrm{m})$.

A medida das dobras cutâneas foi efetuada em triplicata, de forma não consecutiva. Na mensuração, utilizou-se plicômetro da Lange ${ }^{\circledR}$, com precisão de $0,1 \mathrm{~mm}$.

Para os cálculos da Percentagem de Gordura $(\% \mathrm{G})$, foi utilizada a equação de predição proposta por Slaughter et al. ${ }^{26}$. 


\section{Procedimentos}

Inicialmente, entrou-se em contato com os treinadores de variadas equipes de diversificadas modalidades esportivas para explanar os procedimentos e os objetivos do estudo. Após consentimento, marcou-se reunião com cada equipe para dialogar com os atletas e explicar todos os procedimentos éticos da pesquisa.

Em seguida, o estudo foi dividido em dois momentos. Na primeira etapa, apenas um pesquisador ficou responsável pela aplicação dos instrumentos BSQ e EAT-26. Essa cautela foi tomada para que não houvesse diferenças de explicações e influência de outros pesquisadores nas respostas dos questionários autoaplicáveis. Distribuíram-se os questionários no primeiro encontro e o seu preenchimento foi voluntário.

O segundo momento foi destinado à realização das aferições de medidas antropométricas. Os clubes disponibilizaram salas adequadas para as avaliações. Os atletas foram conduzidos, individualmente, para essa sala com o propósito de anular possíveis interferências de colegas de equipe. Ademais, essas medidas foram aferidas pelo mesmo avaliador com objetivo de aumentar a fidedignidade da avaliação. Realizou-se o cálculo do Erro Técnico de Medida (ETM) proposto por Perini et al. ${ }^{27}$, excluindo-se dados com variância maior que $10 \%$.

\section{Análise dos dados}

Utilizaram-se medidas de tendência central (média e desvio-padrão) para as variáveis insatisfação corporal e comportamento alimentar inadequado. Conduziu-se Análise Univariada de Covariância (ANCOVA), utilizando idade, IMC e \% G como covariáveis, no comparo da insatisfação corporal e do comportamento alimentar inadequado entre os estágios de maturação sexual e somática. Para averiguar a razão de chances para insatisfação corporal e o comportamento alimentar inadequado, utilizou-se a regressão logística binária. Nesse teste, as classificações de insatisfação corporal do BSQ foram agrupadas em "insatisfeitos". Aplicou-se a regressão linear múltipla stepwise para determinar a influência que a maturação sexual e somática exercia sobre a insatisfação corporal e o comportamento alimentar. Todos os dados foram tratados no software Statistical Package for the Social Sciences (SPSS) versão 17.0, com nível de significância de 5\%.

RE S U L T A D O S

Os resultados da comparação de insatisfação corporal entre os estágios maturacionais, utilizando a idade, o IMC e o \%G como covariáveis, evidenciaram diferenças estatisticamente significativas $(p<0,05)$. No sexo feminino, essas diferenças estavam presentes na maturação sexual; no masculino, identificou-se diferença entre os estágios de maturação somática (Tabela 2). Não se encontrou diferença de comportamento alimentar entre os diferentes estágios maturacionais.

Somente no sexo masculino, os estágios maturacionais puderam predizer a insatisfação corporal (Tabela 3). Meninos pré-púberes e púberes apresentaram maiores chances de serem insatisfeitos comparados a atletas pós-púberes $(p<0,05)$. Além disso, encontraram-se maiores chances de insatisfação com peso e aparência corporal em atletas masculinos que não haviam chegado ao estirão de crescimento (pré-estirão) quando foram comparados aos adolescentes que já passaram desse evento maturacional $(p<0,05)$.

O modelo de regressão logística simples mostrou associação entre comportamento alimentar inadequado e estágios maturacionais somente no sexo masculino (Tabela 4). Dois achados merecem destaque: meninos pré-púberes apresentaram maiores chances de alimentação desordenada do que atletas pós-púberes $(p<0,05)$, e adolescentes do sexo masculino pré-estirão, em relação à maturação somática, mostraram maiores chances de apresentarem comportamentos alimentares inadequados quando comparados ao grupo pós-estirão $(p<0,05)$. 
Tabela 2. Média e desvio-padrão da insatisfação corporal e do comportamento alimentar inadequado entre diferentes estágios de maturação sexual e somática de jovens atletas. Rio de Janeiro (RJ), Três Rios (RJ), Juiz de Fora (MG), Barbacena (MG), Belo Horizonte (MG), 2011.

\begin{tabular}{|c|c|c|c|c|c|}
\hline \multirow{2}{*}{ Sexo } & \multirow{2}{*}{ Estágio } & \multicolumn{2}{|c|}{ BSQ } & \multicolumn{2}{|c|}{ EAT-26 } \\
\hline & & M & DP & M & DP \\
\hline \multicolumn{6}{|c|}{ Maturação sexual } \\
\hline \multirow[t]{5}{*}{$\mathrm{F}$} & Pré-púbere & 77,22 & 19,47 & 17,38 & 6,02 \\
\hline & Púbere inicial & 96,98 & $9,68^{e}$ & 16,60 & 2,99 \\
\hline & Púbere intermediário & 76,04 & 4,97 & 11,60 & 1,54 \\
\hline & Púbere avançado & 79,00 & $3,64^{\mathrm{e}}$ & 12,55 & 1,12 \\
\hline & Pós-púbere & 77,22 & 5,62 & 17,38 & 1,74 \\
\hline \multirow[t]{5}{*}{ M } & Pré-púbere & 56,60 & 11,88 & 16,24 & 6,93 \\
\hline & Púbere inicial & 65,18 & 4,84 & 16,41 & 2,82 \\
\hline & Púbere intermediário & 63,29 & 1,92 & 11,45 & 1,12 \\
\hline & Púbere avançado & 59,66 & 1,49 & 12,06 & 0,86 \\
\hline & Pós-púbere & 55,87 & 1,95 & 10,34 & 1,13 \\
\hline \multicolumn{6}{|c|}{ Maturação somática } \\
\hline \multirow[t]{3}{*}{$\mathrm{F}$} & Pré-estirão & 45,02 & 21,04 & 5,01 & 6,29 \\
\hline & Estirão & 69,05 & 6,96 & 8,19 & 2,08 \\
\hline & Pós-estirão & 45,02 & 3,43 & 5,01 & 1,02 \\
\hline \multirow[t]{3}{*}{ M } & Pré-estirão & 69,58 & 3,81 & 12,64 & 2,23 \\
\hline & Estirão & 61,33 & 1,72 & 11,23 & 1,01 \\
\hline & Pós-estirão & 56,05 & $1,94^{f}$ & 12,64 & 1,16 \\
\hline
\end{tabular}

${ }^{\mathbf{e}} p<0,05$ em relação ao pós-púbere; ${ }^{\mathfrak{f}} p<0,05$ em relação ao pré-estirão.

BSQ: Body Shape Questionnaire; EAT-26: Eating Attitudes Test; M: Média; DP: Desvio-Padrão; F: Feminino; M: Masculino.

Tabela 3. Razão de chances para insatisfação corporal (categoria de referência: satisfeitos) em jovens atletas divididos por sexo, segundo estágios maturacionais. Rio de Janeiro (RJ), Três Rios (RJ), Juiz de Fora (MG), Barbacena (MG), Belo Horizonte (MG), 2011.

\begin{tabular}{|c|c|c|c|c|}
\hline Sexo & Maturação & Classificação & OR & IC (95\%) \\
\hline \multirow[t]{6}{*}{$\mathrm{F}$} & \multirow[t]{4}{*}{ Sexual } & Pré-púbere & 1,00 & $0,04-20,82$ \\
\hline & & Púbere intermediário & 0,47 & $0,05-14,96$ \\
\hline & & Púbere avançado & 0,88 & $0,01-6,37$ \\
\hline & & Pós-púbere & 0,35 & \\
\hline & \multirow{2}{*}{ Somática } & Estirão & 1,14 & $0,01-20,43$ \\
\hline & & Pós-estirão & 1,30 & $0,64-8,96$ \\
\hline \multirow[t]{5}{*}{ M } & \multirow[t]{2}{*}{ Sexual $^{\star}$} & Pré-púbere & 4,06 & $0,34-4,75$ \\
\hline & & Púbere inicial & 2,16 & $0,63-7,39$ \\
\hline & \multirow[t]{3}{*}{ Somática* } & Pré-estirão & 1,37 & $0,60-3,12$ \\
\hline & & Estirão & 1,17 & $0,67-2,04$ \\
\hline & & Pós-estirão & 1,00 & \\
\hline
\end{tabular}

* $p<0,05$; F: Feminino; M: Masculino; OR: Razão de Chances (odds ratio); IC: Intervalo de Confiança.

Quanto à influência do processo maturacional exercida sobre a insatisfação corporal e o comportamento alimentar inadequado em atletas adolescentes, no sexo feminino, a interação entre maturação sexual e somática explicou em $9 \%$ e $7 \%$ a variância da insatisfação corporal $(p<0,05)$ 
e do comportamento alimentar inadequado $(p<0,05)$, respectivamente. Entre os meninos, a insatisfação corporal pareceu não sofrer influência da maturação biológica. Entretanto, o processo maturacional (somático e sexual) modulou 3\% da variância do comportamento alimentar entre atletas desse sexo $(p<0,05)$ (Tabela 5).
D I S C U S S Ã O

O presente estudo apresentou como premissa analisar a associação entre o processo maturacional, a insatisfação corporal e o comportamento alimentar inadequado segundo o sexo em jovens atletas. Os resultados da presente

Tabela 4. Razão de chances para comportamento alimentar inadequado (categoria de referência: negativo) em jovens atletas divididos por sexo, segundo estágios maturacionais. Rio de Janeiro (RJ), Três Rios (RJ), Juiz de Fora (MG), Barbacena (MG), Belo Horizonte (MG), 2011.

\begin{tabular}{|c|c|c|c|c|}
\hline Sexo & Maturação & Classificação & OR & IC (95\%) \\
\hline \multirow[t]{8}{*}{$\mathrm{F}$} & \multirow[t]{5}{*}{ Sexual } & Pré-púbere & 1,00 & - \\
\hline & & Púbere inicial & 0,25 & $0,01-5,98$ \\
\hline & & Púbere intermediário & 0,16 & $0,00-3,23$ \\
\hline & & Púbere avançado & 0,28 & $0,01-5,01$ \\
\hline & & Pós-púbere & 0,12 & $0,00-2,56$ \\
\hline & \multirow[t]{3}{*}{ Somática } & Pré-estirão & 1,00 & \\
\hline & & Estirão & 1,86 & $0,00-8,95$ \\
\hline & & Pós-estirão & 1,89 & $0,02-7,61$ \\
\hline \multirow[t]{8}{*}{ M } & \multirow[t]{5}{*}{ Sexual ${ }^{*}$} & Pré-púbere & 25,20 & $2,09-30,25$ \\
\hline & & Púbere inicial & 4,50 & $1,34-15,04$ \\
\hline & & Púbere intermediário & 3,05 & $1,40-6,65$ \\
\hline & & Púbere avançado & 2,07 & $0,95-4,47$ \\
\hline & & Pós-púbere & 1,00 & - \\
\hline & \multirow[t]{3}{*}{ Somática* } & Pré-estirão & 2,98 & $1,38-6,42$ \\
\hline & & Estirão & 1,79 & $1,00-3,20$ \\
\hline & & Pós-estirão & 1,00 & \\
\hline
\end{tabular}

*p<0,05; F: Feminino; M: Masculino; OR: Razão de Chances (odds ratio); IC: Intervalo de Confiança.

Tabela 5. Influência do processo maturacional sobre a insatisfação corporal e o comportamento alimentar inadequado em jovens atletas. Rio de Janeiro (RJ), Três Rios (RJ), Juiz de Fora (MG), Barbacena (MG), Belo Horizonte (MG), 2011.

\begin{tabular}{|c|c|c|c|c|c|}
\hline Sexo & Maturação & $\mathrm{R}$ & $\mathrm{R}^{2}$ & $\mathrm{R}^{2}$ ajustado & $p$-valor \\
\hline \multicolumn{6}{|c|}{ Insatisfação corporal } \\
\hline \multirow[t]{3}{*}{ Feminino } & Sexual & 0,10 & 0,010 & 0,001 & 0,29 \\
\hline & Somática & 0,17 & 0,030 & 0,020 & 0,06 \\
\hline & Sexual ${ }^{\star}$ somática & 0,30 & 0,090 & 0,070 & 0,05 \\
\hline \multirow[t]{3}{*}{ Masculino } & Sexual & 0,08 & 0,007 & 0,005 & 0,06 \\
\hline & Somática & 0,05 & 0,030 & 0,001 & 0,22 \\
\hline & Sexual* somática & 0,08 & 0,007 & 0,003 & 0,19 \\
\hline \multicolumn{6}{|c|}{ Comportamento alimentar inadequado } \\
\hline \multirow[t]{3}{*}{ Feminino } & Sexual & 0,10 & 0,010 & 0,003 & 0,25 \\
\hline & Somática & 0,14 & 0,020 & 0,010 & 0,14 \\
\hline & Sexual ${ }^{\star}$ somática & 0,26 & 0,070 & 0,050 & 0,01 \\
\hline \multirow{3}{*}{ Masculino } & Sexual & 0,16 & 0,030 & 0,020 & 0,01 \\
\hline & Somática & 0,15 & 0,020 & 0,020 & 0,01 \\
\hline & Sexual $^{*}$ somática & 0,17 & 0,030 & 0,020 & 0,01 \\
\hline
\end{tabular}


investigação apontaram diferenças de insatisfação corporal em função do estágio maturacional somente para o sexo feminino. Todavia, não foram identificadas diferenças estatisticamente significativas nos escores do EAT-26 segundo os estágios maturacionais. Além disso, o modelo de regressão logística binária evidenciou associação de insatisfação corporal e comportamento alimentar inadequado apenas para o sexo masculino. Ressalta-se, entretanto, que tais achados podem ser fruto das especificidades do público de atletas. Autores salientam que esportistas competitivos costumam apresentar discrepâncias elevadas em relação aos não atletas a respeito do nível de atividade física, da composição corporal e dos traços de personalidade ${ }^{28,29}$. Dessa maneira, a sistematização do treinamento físico pode gerar redução do percentual de gordura ${ }^{28}$, que, por sua vez, pode diminuir o descontentamento com o corpo e consequentemente reduzir a frequência na utilização de métodos patogênicos para controle/perda de peso corporal em atletas ${ }^{17,24}$. Em contrapartida, pesquisadores afirmam que as características de personalidade evidenciadas em atletas podem ser consideradas de risco para o desenvolvimento dos sintomas alimentares patológicos ${ }^{4,17}$.

Os resultados encontrados no sexo feminino evidenciaram que atletas no estágio pós-púbere eram menos insatisfeitas com o corpo do que as meninas púberes (inicial e avançado). Esse achado indica que meninas atletas em estágio maturacional precoce apresentavam maior preocupação com peso e aparência física quando comparadas às esportistas em fases maturacionais mais avançadas. Entretanto, os estágios pubertários, tanto da maturação sexual quanto da somática, não se associaram significativamente com a insatisfação corporal no modelo de regressão logística, e a maturação biológica explicou somente $9 \%$ da variância da insatisfação corporal nesse sexo. Desse modo, esse resultado aponta que o processo maturacional influenciou o descontentamento com o corpo em jovens atletas do sexo feminino, ou seja, cerca de $10 \%$ da modulação de sentimentos/pensamentos negativos a respeito do corpo podem ser explicados pelas alterações morfológicas reguladas pela maturação biológica em meninas esportistas. Sendo assim, os resultados supracitados não corroboram as hipóteses descritas na introdução, com exceção da significância estatística encontrada na regressão linear múltipla. Possivelmente, em atletas do sexo feminino, o processo de maturação biológica não exerça grandes riscos para tal desfecho, o que remete à hipótese de que, nesse público, a insatisfação corporal seja influenciada principalmente por fatores do ambiente esportivo. Segundo De Bruin et al. ${ }^{2}$, Sundgot-Borgen \& Torstveit ${ }^{16}$ e Krentz \& Warchsburger ${ }^{17}$, a cultura atlética é o principal agente negativo para acentuar a preocupação com peso e aparência física entre os esportistas. Entretanto, deve-se salientar que, por se tratar de jovens que residem em país ocidental, é possível que meninas atletas, independentemente da fase da adolescência em que estejam, possam internalizar o ideal de magreza corporal, o que as torna mais susceptíveis à insatisfação corporal ${ }^{2,3}$.

No sexo masculino, os achados mostraram que atletas que ainda não haviam atingido o PVA eram mais insatisfeitos com o corpo do que os que já haviam passado por esse período. Portanto, pode-se interpretar que meninos esportistas biologicamente mais jovens eram mais descontentes com peso e aparência corporal do que os atletas adolescentes biologicamente mais avançados. Além disso, identificou-se que meninos nos estágios iniciais de maturação biológica apresentaram maiores chances de insatisfação corporal em relação aos atletas em estágios avançados, conforme apontado nas hipóteses do estudo. No entanto, acredita-se que a maior razão de chances para insatisfação corporal encontrada em pré-púberes e púberes iniciais não foi influenciada somente pelos estágios maturacionais. Estima-se também que esses sujeitos estejam mais susceptíveis a adaptações psicológicas negativas advindas de cobranças de treinadores, familiares e amigos devido à otimização do rendimento esportivo. Denoma et al. ${ }^{29}$ salientam que atletas 
mais jovens podem apresentar maior frequência de adaptações psicológicas negativas quando suas performances são questionadas. Nesse caso, atletas pré-púberes e indivíduos no início da puberdade podem compensar essa cobrança na aquisição de comportamentos compensatórios com o propósito de perda de peso ou diminuição do perfil lipídico, podendo desencadear os transtornos alimentares ainda em idade relativamente precoce. Por fim, a maturação biológica não exerceu influência sobre a insatisfação corporal nesse sexo. Dessa maneira, esse achado aponta que as alterações de áreas corporais reguladas pelo processo maturacional não explicaram a variância do descontentamento com o corpo em jovens atletas do sexo masculino. Portanto, mais investigações necessitam ser realizadas a fim de esclarecer melhor esse tópico.

Em relação ao comportamento alimentar inadequado no sexo feminino, os resultados obtidos não apresentaram diferenças estaticamente significativas entre os estágios maturacionais. Além do mais, esses estágios não se associaram com o comportamento alimentar no modelo de regressão logística. Sendo assim, parece que as atletas adolescentes do sexo feminino podem apresentar riscos para o comportamento alimentar inadequado, independentemente do seu estágio maturacional. Esses achados, portanto, fogem das tendências formuladas na introdução da presente investigação. Apesar de existirem hipóteses a respeito da possível influência da maturação biológica sobre atitudes alimentares em jovens ${ }^{2}$, o processo maturacional modulou apenas 9\% da variância do comportamento alimentar inadequado: assim como evidenciado para a insatisfação corporal, parece que o processo maturacional influencia aproximadamente $10 \%$ dos hábitos alimentares deletérios à saúde em meninas atletas. Provavelmente, a grande prevalência de hábitos alimentares não saudáveis encontrados na população de atletas femininas ${ }^{15}$ seja explicada por outros fatores. Um deles pode ser o tipo de esporte, pois, segundo Krentz \& Warschburger ${ }^{17}$, modalidades que priorizam a estética corporal magra e os esportes que dividem categorias por classe de peso apresentam maiores riscos para tal desfecho. Além disso, o nível competitivo também parece exercer grande influência no aparecimento desses comportamentos ${ }^{29}$.

Referindo-se aos resultados encontrados em relação ao sexo masculino para o comportamento alimentar inadequado, evidenciou-se que esses hábitos em atletas adolescentes foram semelhantes nos diferentes estágios maturacionais. Esses achados não corroboram as hipóteses levantadas no início deste estudo. Em contrapartida, identificou-se que, com o avanço pubertário, houve diminuição das chances para alimentação desordenada nesse sexo. Portanto, esses achados apontam que meninos atletas mais avançados biologicamente estavam menos vulneráveis ao desencadeamento de atitudes alimentares inapropriadas. Apesar da associação significativa encontrada no modelo de regressão logística binária entre estágios maturacionais e comportamento alimentar, o processo maturacional na regressão linear múltipla stepwise explicou somente 3\% da modulação dessa variável.

A alimentação desordenada em homens atletas pode ser mais bem explicada por outros fatores. Autores afirmam que o tipo de esporte ${ }^{17}$, principalmente os que apresentam divisões por classe de peso $0^{9,10}$, são precursores de hábitos alimentares não saudáveis nesse sexo. Segundo Filaire et al. ${ }^{10}$, a indução de vômitos e a restrição alimentar são práticas alimentares comuns entre esportistas de judô e tae kwon do.

De modo geral, acredita-se que tanto a insatisfação corporal quanto o comportamento alimentar inadequado sejam fenômenos que sofram influência de diversos fatores, principalmente no contexto esportivo. Influências culturais, familiares e midiáticas a que são expostos os adolescentes, somadas às cobranças por melhores resultados e controle de peso corporal, podem contribuir para que os atletas sejam mais susceptíveis a riscos psicológicos. O ambiente esportivo é bastante complexo para se acreditar que somente algumas variáveis possam explicar o fenômeno. 
O presente estudo procurou preencher uma lacuna do conhecimento existente na literatura. No entanto, esta pesquisa apresentou limitações: a principal foi utilizar instrumentos autoaplicáveis, pois indivíduos podem não responder com fidedignidade a essas ferramentas de pesquisa. Os resultados, portanto, podem não refletir a realidade do contexto avaliado, visto que o resultado final é fruto de respostas subjetivas. Contudo, este trabalho mostra importantes evidências a respeito da influência e dos riscos que o processo maturacional pode exercer sobre a insatisfação corporal e o comportamento alimentar inadequado em atletas adolescentes, acrescentando à literatura algumas questões relevantes que até então não haviam sido levantadas.

\section{O N CLUS Ã O}

Os resultados encontrados no estudo permitem considerar que o processo maturacional exerceu pouca influência sobre a insatisfação corporal e o comportamento alimentar inadequado em ambos os sexos. Ademais, os estágios de maturação biológica associaram-se com os desfechos somente no sexo masculino. Além disso, tanto atletas femininas quanto masculinos apresentaram maiores índices de insatisfação corporal em estágios pubertários iniciais quando comparados aos finais.

Sugere-se que sejam realizadas mais pesquisas em população com características semelhantes à deste estudo, englobando outras modalidades esportivas e averiguando se o tipo de esporte exerce influência sobre a insatisfação corporal e o comportamento alimentar de risco para transtornos alimentares. Além disso, encorajam-se estudos que avaliem a influência de outros fatores, como, por exemplo, grau de comprometimento psicológico ao exercício, etnia, níveis competitivos e econômicos sobre a insatisfação corporal e hábitos alimentares inapropriados de jovens atletas brasileiros.

Programas de avaliação e orientação tanto psicológica quanto nutricional são necessários no âmbito esportivo: essas iniciativas devem ser desenvolvidas com o caráter de prevenção e de detecção de doenças, como os transtornos alimentares em atletas.

\section{COLABORADORES}

LS FORTES coletou os dados, redigiu o artigo e realizou o tratamento dos dados. SS ALMEIDA revisou o artigo e participou das análises estatísticas. MEC FERREIRA revisou o artigo e orientou todo o trabalho.

\section{REFERÊ NCIAS}

1. World Health Organization. Development of a WHO growth reference for school-aged children and adolescents. Bull World Health Organ. 2007; 85(9):660-7. doi: 10.2471/BLT.07.04349.

2. De Bruin AP, Oudejans RRD, Bakker FC. Dieting and body image in aesthetic sports: a comparison of dutch female gymnnasts and non-aesthetic sport partcipants. Psychol Sport Exerc. 2007; 8(4): 507-20. doi: 10.1016/j.psychsport.2006.10.002.

3. Haase AM. Weight perception in female athletes: associations with disordered eating correlates and behavior. Eat Behaviors. 2011; 12(1):64-7. doi: 10.1 016/j.eatbeh.2010.09.004.

4. Schaal K, Tafflet M, Nassif H, Thibault V, Pichard C, Alcotte $M$, et al. Psychological balance in high level athletes: gender-based differences and sportspecific patterns. Psychopathol High Level Sport. 2011; 6(5):1-9. doi: 10.1371/journal.pone.001 9007.

5. Siervogel RM, Demerath EW, Schubert C, Remsberg KE, Chumlea WC, SUN S, et al. Puberty and body composition. Horm Res. 2003; 60(1):36-45. doi: 10.1159/000071224.

6. Mirwald RL, Baxter-Jones ADG, Bailey DA, Beunen GP. An assessment of maturity from anthropometric measurements. Med Sci Sport Exerc. 2002; 34(4): 689-694. doi: 01 95-913 i/02/3404-0689.

7. Hausenblas HA, Downs DS. Comparison of body image between athletes and nonathletes - a meta - analytic Review. J Appl Sports Psych. 2001; 13(2):323-39. doi: 1041-3200/01.

8. McGehee TMT, Green JM, Leeper JD, Leaver-Dunn D, Richardson M, Bishop PA. Body image, anthropometric measures, and eating-disorder prevalence in auxiliary unit members. J Athletic Training. 2009; 44(4):418-26. 
586 | LS FORTES et al

9. Picket TC, Lewis RJ, Cash TF. Men, muscles, and body image: comparisons of competitive bodybuilders, weight trainers, and athletically active controls. Br J Sports Med. 2005; 39(2):217-22. doi: 10.1136/bjsm.2004.012013.

10. Filaire E, Rouveix A, Pannafieux C, Ferrand C. Eating attitudes perfectionism and body-esteem of elite male judoists and cyclists. J Sports S Med. 2007; 6(1):50-7.

11. Johnson C, Crosby R, Engel S, Mitchell J, Powers P, Wittrock D, et al. Gender, ethnicity, self-esteem and disordered eating among college athletes. Eat Behav. 2004; 5(1):147-56. doi: 10.1016/j.eatbeh. 2004.01.004.

12. Lepage $\mathrm{ML}, \mathrm{Crowther} \mathrm{JH}$. The effects of exercise on body satisfaction and affect. Body Image. 2010; 7(1):124-30. doi: 10.1016/j.bodyim.2009.12.002.

13. Bonci $C M$, Bonci $\sqcup$, Granger LR, Johnson CL, Malina RM, Milne LW, et al. National athletic trainers' association position statement: preventing, detecting, and managing disordered eating in athletes. J Athletic Training. 2008; 43(1):80-108.

14. Bighetti F, Santos CB, Santos JE, Ribeiro RPP. Tradução e avaliação do eating attitudes test em adolescentes do sexo feminino de Ribeirão Preto, São Paulo. J Bras Psiq. 2004; 53(6):339-46.

15. Torstveit MK, J Sundgot-Borgen. The female athlete triad exists in both elite athletes and controls. Med Sc Sports Exerc. 2005, 37(1):1449-59.

16. Sundgot-Borgen J, Torstveit MK. Prevalence of disorders in elite athletes in higher than in the general population. Clin J Sport Med. 2004; 14(1): 25-32. doi: 10.1249/01.mss.0000177678.7304 1.38.

17. Krentz EM, Warschburger P. Sports-related correlates of disordered eating in aesthetic sports. Psychol Sport Exerc. 2011, 44(3):315-21. doi: 10.1 016/j.psychsport.2011.03.004.

18. Scherer FC, Martins CB, Pelegrini A, Matheus SC, Petroski EL. Imagem corporal em adolescentes: associação com a maturação sexual e sintomas de transtornos alimentares. Rev Bras Psiquiatr. 2010; 59(3):198-202. doi: 10.1590/S0047-2085201000 0300005

19. Baxter-Jones D, Eisenmann JC, Sherar LB. Controlling for maturation in Pediatric exercise science. Pediatr Exerc Sci. 2005; 17(1):18-30.
20. Perini TA, Vieira RS, Vigário OS, Oliveira GL, Ornellas JS, Oliveira FP. Transtorno do comportamento alimentar em atletas de elite de nado sincronizado. Rev Bras Med Esporte. 2009; 15(1):54-7. doi: 10.1 590/S1517-86922009000100012.

21. Malina R, Bouchard C, Bar-Or O. Crescimento, maturação e atividade física. São Paulo: Phorte; 2009.

22. Matsudo SMM, Matsudo VKR. Validade da auto avaliação na determinação da maturação sexual. Rev Bras Ciênc Mov. 1991; 5(2):18-35.

23. Conti MA, Cordás TA, Latorre MRDO. Estudo de validade e confiabilidade da versão brasileira do Body Shape Questionnaire para adolescentes. Rev Bras Saúde Mater Infant. 2009; 9(3):331-8. doi: 10.1590/S1519-38292009000300012.

24. Fortes LS, Amaral ACS, Conti MA, Cordás TA, Ferreira MEC. Qualidades psicométricas do eating attitudes test para o adolescente. Psicol Reflex Crít. (no prelo).

25. The Internacional Society for Advancement for Kineanthropometry. Camberra: National Library of Australia; 2001.

26. Slaughter MH, Lohman TG, Boileau R, Hoswill CA, Stillman RJ, Yanloan MD, et al. Skinfold equations for estimation of body fatness in children and youth. Hum Biol. 1988; 60(5):709-23.

27. Perini TA, Oliveira GL, Ornellas JS, Oliveira FP. Cálculo do erro técnico de medição em antropometria. Rev Bras Med Esporte. 2005; 11(1):81-5. doi: 10.15 90/S1517-86922005000100009.

28. Fortes LS, Ferreira MEC. Comparação da insatisfação corporal e do comportamento alimentar inadequado em atletas adolescentes de diferentes modalidades esportivas. Rev Bras Educ Fis Esporte. 2011; 25(4):707-16. doi: 10.1590/\$1807-5509201 1000400014.

29. Denoma JMH, Scaringi V, Gordon KH, Van Orden $K A$, Joiner TE. Eating disorder symptoms among undergraduate varsity athletes. Club athletes, independent exercisers and nonoexercises. Int J Eat Disord. 2009; 12(1):47-53. doi: 10.1002/eat.2 0560. 